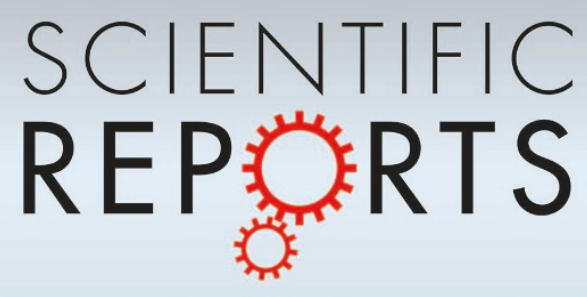

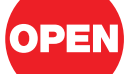

SUBJECT AREAS:

PHYSICS

CONDENSED-MATTER PHYSICS

MATERIALS SCIENCE

GRAPHENE

Received

23 July 2012

Accepted

2 October 2012

Published

14 November 2012

Correspondence and requests for materials should be addressed to J.L. (jinglu@pku.edu.

$\mathrm{cn})$

\section{Tunable and sizable band gap in silicene by surface adsorption}

\author{
Ruge Quhe ${ }^{1,2}$, Ruixiang Fei', Qihang Liu', Jiaxin Zheng ${ }^{1,2}$, Hong Li' ${ }^{1}$, Chengyong Xu' ${ }^{1}$, Zeyuan $\mathrm{Ni}^{1}$, \\ Yangyang Wang' ' Dapeng $\mathrm{Yu}^{\prime}$, Zhengxiang Gao' \& Jing Lu'
}

${ }^{1}$ State Key Laboratory of Mesoscopic Physics and Department of Physics, Peking University, Beijing 100871, P. R. China, ${ }^{2}$ Academy for Advanced Interdisciplinary Studies, Peking University, Beijing 100871, P. R. China.

Opening a sizable band gap without degrading its high carrier mobility is as vital for silicene as for graphene to its application as a high-performance field effect transistor (FET). Our density functional theory calculations predict that a band gap is opened in silicene by single-side adsorption of alkali atom as a result of sublattice or bond symmetry breaking. The band gap size is controllable by changing the adsorption coverage, with an impressive maximum band gap up to $0.50 \mathrm{eV}$. The $a b$ initio quantum transport simulation of a bottom-gated FET based on a sodium-covered silicene reveals a transport gap, which is consistent with the band gap, and the resulting on/off current ratio is up to $10^{8}$. Therefore, a way is paved for silicene as the channel of a high-performance FET.

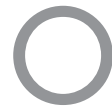

wing to its extremely high carrier mobility, graphene has attracted enormous attention since its discovery in $2004^{1}$. However, pure graphene is not suitable for effective field effect transistor (FET) operating at room temperature due to its zero band gap. Opening a sizable band gap without degrading the electronic properties is critical for the application of graphene in nanoelectronics and is probably one of the most important and tantalizing research topics of the graphene community. Any successor to silicon metal-oxide-semiconductor FET (MOSFET) that is used in complementary MOS-like logic must have a sizeable band gap of $0.4 \mathrm{eV}$ or more and an on-off current ratio between $10^{4}$ and $10^{72}$. Unfortunately, the opened band gap without degrading the electronic properties in graphene (via a vertical electric field, molecular surface adsorption, or hexagonal boron nitride (h-BN) as a substrate) is usually smaller than $0.34 \mathrm{eV}^{3-8}$, and the observed on-off current ratio at room temperature is no more than $100^{9}$. Silicene, graphene analogue for silicon, is predicted to be a zero band gap semiconductor with a Dirac cone as observed in graphene ${ }^{10,11}$. The synthesis of silicene on the Ag surface ${ }^{12-17}$ or zirconium diboride thin film ${ }^{18}$ has already been reported in several experiments. Recently, the linear band dispersion near the Fermi level has been observed in a silicene sample on $\mathrm{Ag}(111)$ surface by angle-resolved photoemission spectroscopy (ARPES), unambiguously verifying the existence of silicene ${ }^{12}$. With its massless Dirac Fermions character, extremely high carrier mobility is expected in silicene as in graphene. Therefore, silicene is a promising material for high-speed switching devices. Apparently, pure silicene cannot directly be used for effective FETs either because of the same cause as graphene ${ }^{19}$.

Although covalent functionalization ${ }^{20-22}$ or epitaxial growth in the form of nanoribbon ${ }^{15,16} \mathrm{can}$ open a sizable band gap in silicene, the electronic properties are destructed, and the carrier mobility is inevitably decreased to a large extent, as happened in graphene ${ }^{23,24}$. Therefore, opening a sizable and tunable band gap of silicene without degrading the electronic properties is equally highly desired for high-performance silicene FET devices. It is proposed that a vertical electric field can open a tunable band gap in silicene without degrading the electronic properties ${ }^{19}$. However, the maximum opened band gap by the experimentally accessible electric field is just $0.13 \mathrm{eV}$. Moreover, a single-gated device is desirable from the device point of view ${ }^{25}$. Very recently, it is reported that silicene that epitaxial grown on Ag surface or zirconium diboride thin film can open a band gap of about 0.6 or $0.3 \mathrm{eV}$, respectively ${ }^{12,18}$. However, $\mathrm{Ag}$ is an metal substrate, which is undesired in a FET device, and the calculated dispersion relation near the band gap for silicene on zirconium diboride thin film is very flat, which will lead to a rather large carrier effective mass and greatly decrease the carrier mobility. Besides, the size of the band gap of silicene opened by the interaction with substrate is difficult to tune.

In this Article, based on the density functional theory (DFT) calculations we propose that the band gap of silicene can be opened without degrading the electronic properties by surface adsorption of alkali metal (AM). The band gap is tunable by controlling the coverage of AM. Most intriguingly, the maximum band gap is up to $0.50 \mathrm{eV}$ by adsorption of $\mathrm{AM}$ atoms, which meets the band gap requirement of high-performance FETs. 
Subsequently, a bottom-gated FET device based on a sodium-covered silicene monolayer is simulated by the $a b$ initio transport calculations. A sizable transport gap and a high on/off current ratio $\left(\sim 10^{8}\right)$ are available in this device, suggestive of the great potential of silicene as the channel of a high-performance FET.

\section{Results}

The supercell model consists of an $m \times m$ primitive silicene cell $(m=$ $1, \sqrt{3}, 2,3$, and 4$)$ and an $\mathrm{AM}$ atom $(\mathrm{AM}=\mathrm{Li}, \mathrm{Na}, \mathrm{K}, \mathrm{Rb}$, and $\mathrm{Cs})$ on the top. The AM-covered silicene is denoted as $\mathrm{AMSi}_{\mathrm{n}}$, where $n$ is the number of the silicon atom per supercell. We define the coverage $N$ of the AM on silicene as the ratio of the number between the AM and $\mathrm{Si}$ atoms per supercell, and one has $N=1 / n$. The in-plane lattice constant of silicene is taken from that of previous works, $a=b=3.866$ $\AA^{10,26,27}$. Along the $z$-direction perpendicular to the silicene plane, the lattice constant of $30 \AA$ is used.

Geometry and stability. Of all the examined adsorption sites, the $\mathrm{AM}$ atoms favor sitting above the hexagonal ring center of silicene and have the highest sixfold coordination (Fig. 1). Such a sixfold coordination configuration of the AM atom has been reported to be favorable in a AM-covered graphene ${ }^{28}$. A metastable state, in which the AM atoms all sit above the bottom $\mathrm{Si}$ atom, is available (Supplementary Fig. S1). The energy differences between these two states increase from 0.06 to $0.21 \mathrm{eV}$ with the decreasing coverage, which are larger than the room temperature $(\sim 0.026 \mathrm{eV})$ and thus ensure the thermodynamically stability and reliable operation if the AM-covered silicene is fabricated as the FET channel.

As shown in Supplementary Fig. S2, the silicene buckling $\left(d_{0}\right)$ increases from 0.46 to $0.49 \sim 0.77 \AA$ after the AM adsorption, weakly dependent on the AM species and the coverage. On the contary, the distance of the AM atom to the silicene top-surface $\left(d_{1}\right)$ singnificantly ranges from 1.3 to $2.8 \AA$ with the increasing atomic radius of AM, but nearly independent of the coverage $N$.

We define the adsorption energy of the AM atom on silicene as

$$
E_{\mathrm{a}}=E_{\mathrm{Si}}+E_{\mathrm{AM}}-E_{\mathrm{Si}+\mathrm{AM}},
$$

where $E_{\mathrm{Si}}, E_{\mathrm{AM}}$ and $E_{\mathrm{Si}+\mathrm{AM}}$ are the relaxed energy for silicene, the isolated AM atom, and the combined system, respectively. As shown
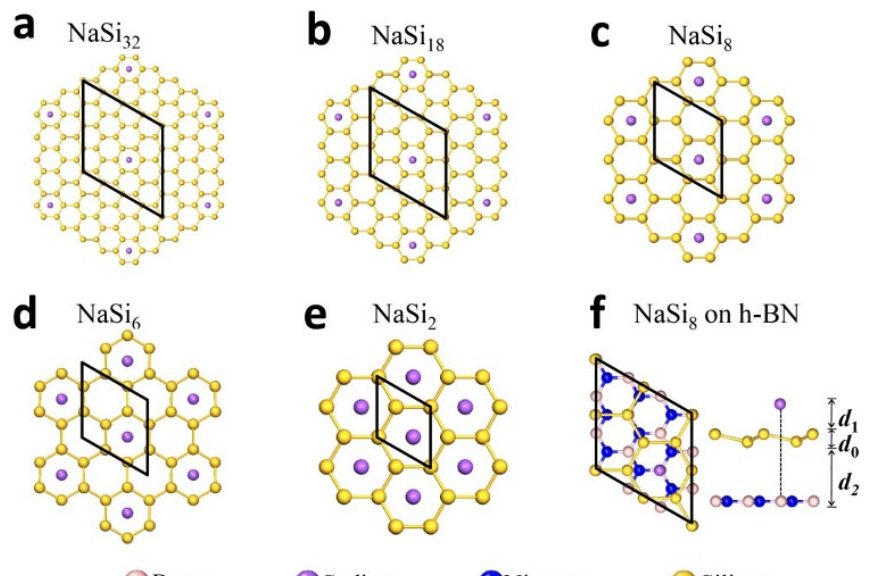

Boron

Sodium

ONitrogen

OSilicon

Figure $1 \mid$ Structures of the Na-covered $\boldsymbol{m} \times \boldsymbol{m}$ silicene supercell. (a-e) The adsorption coverage is $N=3.1 \%, 5.6 \%, 12.5 \%, 16.7 \%$, and $50.0 \%$, respectively, for $m=4,3,2, \sqrt{3}$, and 1 . The rhombi plotted in black line represent the supercells at different coverages. (f) Top and side views of an $\mathrm{Na}$-covered silicene supercell over the h-BN sheet with $N=12.5 \% . d_{0}$ : the silicene buckling; $d_{1}$ : the distance between $\mathrm{Na}$ and the top-surface silicene; $d_{2}$ : the distance between $\mathrm{h}-\mathrm{BN}$ and the bottom-surface of silicene. in Fig. 2a, the $E_{\mathrm{a}}$ values are positive with magnitudes of $0.57 \sim$ $2.92 \mathrm{eV}$ per AM atom, showing that the AM one-side adsorption on silicene layer is always strongly or moderately exothermic. $E_{\mathrm{a}}$ tends to reduce with the increasing $N$. One cause is that the electrostatic attraction between the AM atom and silicene is reduced with the increasing $N$, as a result of the reduced charge transfer $Q$ from the AM cation and silicene (Fig. 2b). Another cause is the repulsion between the AM cations and silicene increases with the increasing $N$. We notice that although the transferred $Q$ from Li to silicene is apparently smaller than those from other AM atoms, $E_{\mathrm{a}}$ of $\mathrm{Li}$ on silicene is comparable with those of other AM atoms on silicene. This suggests an evident 'covalent component' of Li-Si interaction besides the ionic contribution ${ }^{29}$.

Electronic structure. Given the same coverage, silicene covered by different AM species share similar electronic structures. We select Na-covered silicene as a representative and show its electronic structure at different coverages in Fig. 3. Even at the smallest coverage of $N=3.1 \%$, a small direct band gap of $0.04 \mathrm{eV}$ is opened between the $\pi$ and $\pi^{*}$ states while the Dirac cone shape is almost preserved (Fig. 3a). Due to the heavy electron doping, the Fermi level $\left(E_{f}\right)$ is elevated by $0.50 \mathrm{eV}$ with respect to the valence band maximum (VBM). The band gap at the Dirac point is increased monotonically to $0.08,0.17,0.30$, and $0.45 \mathrm{eV}$ at $N=5.6,12.5,16.7$, and $50 \%$, respectively (Fig. 3b-3e). Notably, both of the two original Dirac points $K_{\mathrm{s}}$ and $K_{\mathrm{S}}^{\prime}$ in silicene are folded to the $\Gamma$ point of the reduced first Brillouin zone (BZ) at $N=5.6 \%(\sqrt{3} \times \sqrt{3}$ supercell) and $16.7 \%$ ( $3 \times 3$ supercell), as shown in Fig. $3 \mathrm{~b}$ and $3 \mathrm{~d}$, respectively. The opened maximum band gap has already met the requirement of $0.4 \mathrm{eV}$ for practical FETs operation. At $N=12.5$ and $50 \%$, there is a band across $E_{f}$ around the $\Gamma$ point, which mainly originates from the

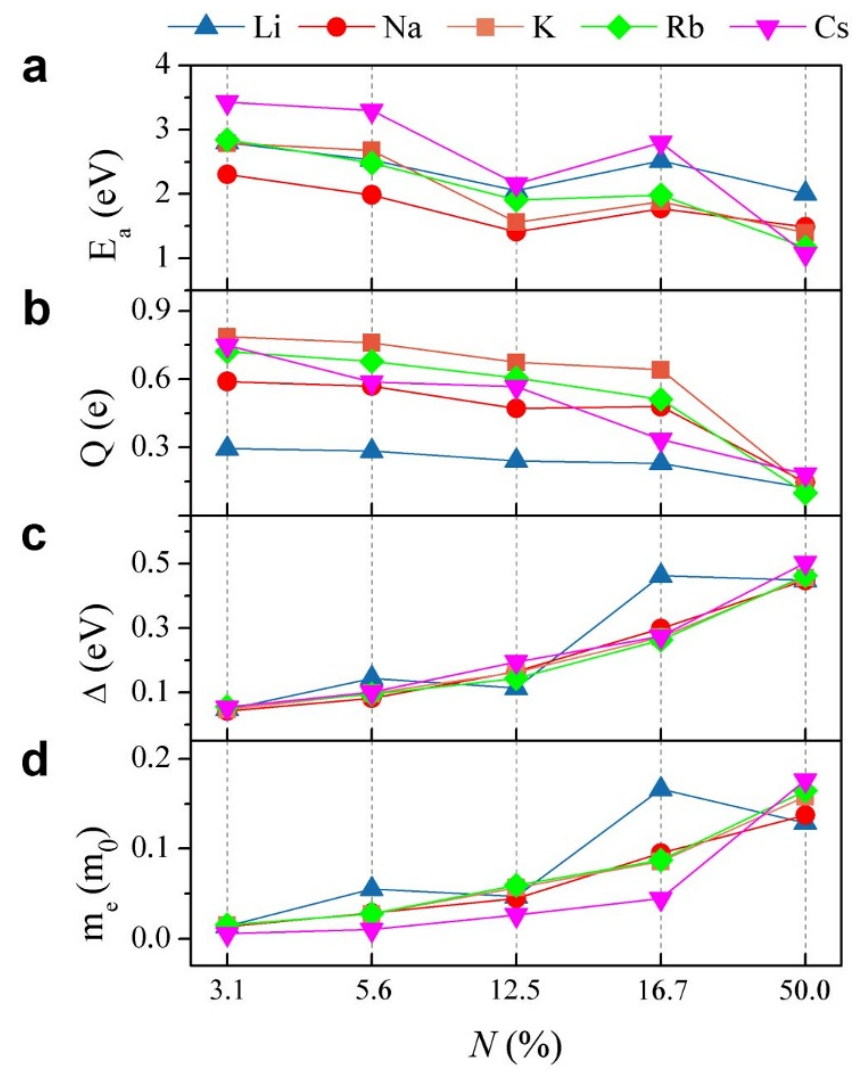

Figure $2 \mid$ (a) Adsorption energy, (b) Mulliken charge transferred from the adatom to silicene, (c) band gap, and (d) effective mass of electrons of the AM-covered silicene as a function of the coverage $N$. Lines connecting symbols are guides to the eye. 
a

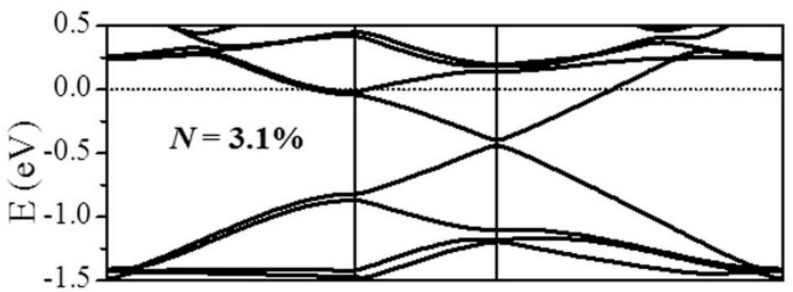

b

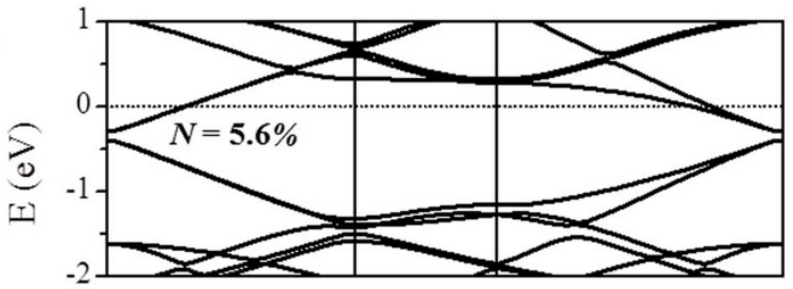

C

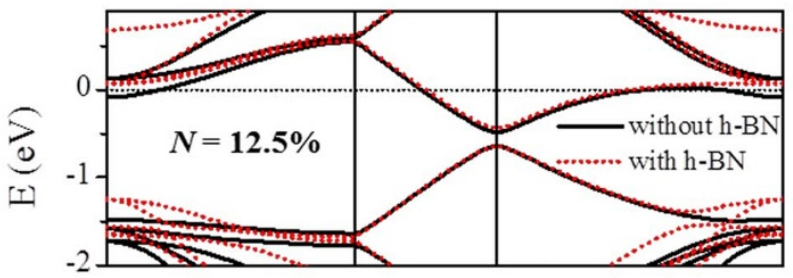

d

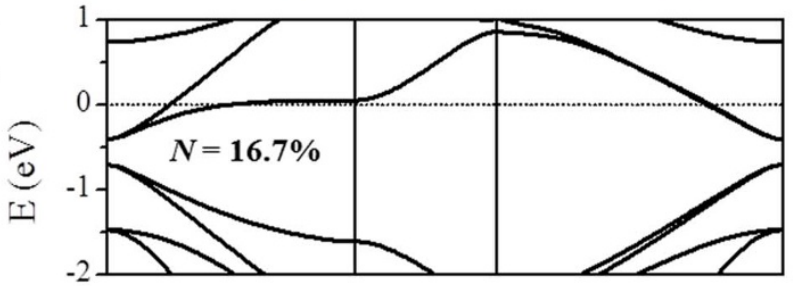

e

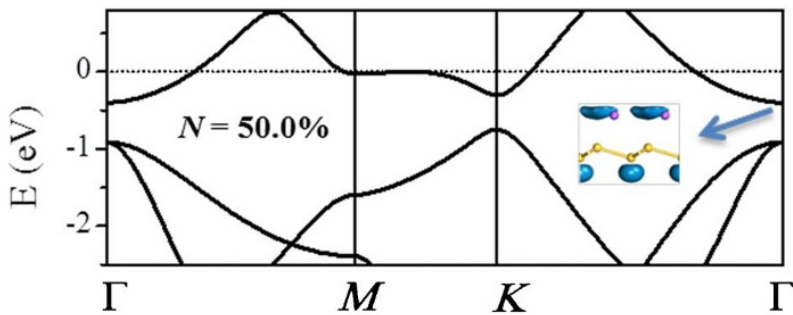

Figure $3 \mid$ Band structures of the Na-covered silicene at different coverages. The Dirac point in (b) and (d) is folded to the $\Gamma$ point due to the reduction of the first Brillouin zone. The red dashed-line in (c) stands for the band structure of the $\mathrm{Na}$-covered silicene with an h-BN buffer layer. Inset of (e): Orbital of the conduction band bottom at the $\Gamma$ point. The Fermi level is set to zero.

broaden outmost $3 s$ valence orbital of the adsorbed $\mathrm{Na}$ atoms (The inset of Fig. 3e).

Fig. $2 \mathrm{c}$ shows the band gap change with the coverage $N$ of the five kinds of the AM-covered silicene. Given a coverage $N$, the band gaps induced by different AM species adsorption are similar except for the $\mathrm{LiSi}_{9}$ and $\mathrm{LiSi}_{6}$ monolayers, the latter of which has an extraordinary large band gap of $0.46 \mathrm{eV}$, compared with a value of about $0.2 \mathrm{eV}$ for other AM-covered silicene at this coverage. The band gaps of all the five kinds of AM-covered silicene monolayers increase generally with the increasing coverage, with values ranging from 0.04 to $0.50 \mathrm{eV}$.

It has been established that a vertical electric filed can induce a band gap in silicene because it breaks the sublattice symmetry in silicene ${ }^{19}$. The AM adsorption leads to a charge transfer between AM and silicene, which builds a perpendicular electric field in silicene. This perpendicular electric field breaks the sublattice symmetry and thus opens a band gap in silicene. The asymmetry between the two sublattices increases with the increasing coverage, and the band gap is correspondingly increased. We should notice a similar phenomenon and band gap opening mechanism in bilayer graphene. Both experiments ${ }^{4,8,25}$ and theoretical calculations ${ }^{5,30}$ reveal that a band gap can be opened in bilayer graphene via single-side adsorption of metal atoms or acceptor/donor molecules because a vertical electric field is built in bilayer graphene due to a charge transfer occurs between the adsorbate and bilayer graphene and it breaks the inversion symmetry of bilayer graphene. The size of the band gap can be controlled by changing the adsorption concentration. Besides the breaking of sublattice symmetry, we point out that the breaking of the bond symmetry also contributes to the band gap opening in the AM-covered silicene. Let us recall its single layer graphene counterpart. When adsorbed on single layer graphene, the Li atoms are also located above the hexagon center of single layer graphene (notated as $\mathrm{LiC}_{\mathrm{n}}$ ), just like the case happened in silicene. If and only if in some certain coverages $(n=3 x, x$ is an arbitrary integer) and the corresponding supercell take forms like $\sqrt{3} \times \sqrt{3}$, $3 \times 3$, etc. (The lattice constant is their integral multiple), the bond symmetry is broken, resulting in a Kekule ordering. Finally, a band gap is created in the $\mathrm{LiC}_{3 x}$ monolayer though the sublattice symmetry of single layer graphene is well preserved ${ }^{31}$.

In an $\mathrm{AMSi}_{\mathrm{n}}$ monolayer, when $n=3 x$, the second band gap opening mechanism also works. It is convenient to interpret this mechanism using the tight-binding model with the Hamiltonian

$$
H=\sum_{i} \varepsilon_{i} c_{i}^{\dagger} c_{i}-\sum_{\langle i j\rangle} t_{i j} c_{i}^{\dagger} c_{j},
$$

where $c_{i}^{\dagger}$ and $c_{i}$ are the fermionic creation and annihilation operators on site $i, \varepsilon_{i}$ the on-site energy, and $t_{\mathrm{ij}}$ the hopping amplitudes. Following this model, we can divide the effects of AM-adsorption into two parts:

1) Breaking of sublattice symmetry. The on-site energies $\varepsilon_{\mathrm{i}}$ are equal in the pure silicene. Due to the charge transfer between
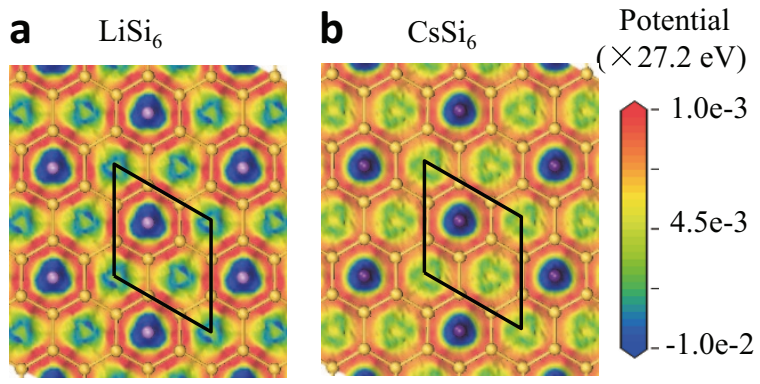

c
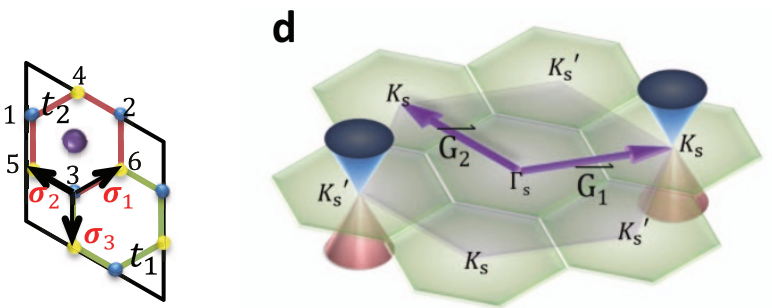

Figure $4 \mid$ Kekulé structures of AM-covered silicene. (a-b) Electrostatic potential distributions in $\mathrm{Li}$ and Cs-covered silicene with $N=16.7 \%$. The rhombi plotted in black line represent the supercells. (c) Schemetic model of the $\mathrm{AMSi}_{6}$ supercell. $t_{1}$ and $t_{2}$ are hopping parameters. Top and bottom silicene sublattices are colored yellow and blue, respectively. The numbers are labeling of the atom sites. (d) The $k$-space of the $\mathrm{AMSi}_{6}$ supercell. The Brillouin zones of the pure silicene and the $\mathrm{AMSi}_{6}$ monolayer are colored purple and green respectively. The $K$ and $K$ Dirac points of silicene can reach each other via $\overrightarrow{\mathrm{G}_{1}}, \overrightarrow{\mathrm{G}_{2}}$ (the unit vectors of the reciprocal lattice of the $\mathrm{AMSi}_{6}$ monolayer) or their linear combination. 
AM and silicene, a perpendicular electric field is built in silicene. Because there is buckling in silicene, the Coulomb fields felt by the top and bottom sublattices are different, resulting to a split of $\varepsilon_{\mathrm{i}}$. We notate the on-site energy of the two sublattices as $\varepsilon_{1}$ and $\varepsilon_{2}$, respectively. The eigen function can be approximately expressed as a linear combination of the normalized $p_{z}$ orbitals of Si atoms. We consider the most simple case of $n=2$ of the AMSi ${ }_{n}$ monolayer, and the Hamiltonian is given by

$$
H=\left(\begin{array}{cc}
\varepsilon_{1} & t \sum_{i=1}^{3} e^{i k \sigma_{i}} \\
t^{*} \sum_{i=1}^{3} e^{-i k \sigma_{i}} & \varepsilon_{2}
\end{array}\right)
$$

where the vectors $\boldsymbol{\sigma}_{i}$ connect the two sublattices of silicene (Fig. 4c). In the Dirac points $\left(t \sum_{i=1}^{3} e^{i k \sigma_{i}}=0\right)$, the opened band gap can be written as

$$
\Delta=\left|\varepsilon_{1}-\varepsilon_{2}\right|
$$

Only if $n \neq 3 x$, the band gap always can be expressed as the function of the on-site energy $\varepsilon_{\mathrm{i}}$. The $\varepsilon_{i}$ discrepancy between the two sublattices is also responsible for the band gap opening of silicene under an external vertical electric field ${ }^{19}$. Apparently, the AM adsorption on the one side of silicene is equivalent to application of a vertical electric field $E_{\mathrm{z}}(x, y)$ with periodic distribution on the silicene plane.

2) Breaking of bond symmetry. Apart from the diagonal terms, there are off-diagonal terms in the Hamiltonian that affect the electronic structure. When $n=3 x$, there is a Kekulé modulation of the hopping amplitudes $t_{\mathrm{ij}}$. In pure silicene, $t_{\mathrm{ij}}$ is a uniform value $(=t)$. As shown in Fig. $4 \mathrm{a}$ and $4 \mathrm{~b}$, the potential of bonds in the hexagon with the AM atoms is quite different from those without them. As a result, the bond symmetry is disturbed, and the nearest-neighbor hopping parameter is split into two different values $t_{1}$ and $t_{2}$. Take the AMSi $\mathrm{A}_{6}$ monolayer as an example (Fig. 4c). Considering their distance respected to the AM atom, we define the on-site energies of the top and bottom Si atoms as $\varepsilon_{1}$ and $\varepsilon_{2}$ respectively. Since the Dirac points of silicene are folded to the $\Gamma$ point of the supercell, the eigenstates and eigenvectors can be simplified as:

$$
\left(\begin{array}{cccccc}
\varepsilon_{1} & 0 & 0 & t_{2} & t_{2} & t_{1} \\
0 & \varepsilon_{1} & 0 & t_{2} & t_{1} & t_{2} \\
0 & 0 & \varepsilon_{1} & t_{1} & t_{2} & t_{2} \\
t_{2}^{*} & t_{2}^{*} & t_{1}^{*} & \varepsilon_{2} & 0 & 0 \\
t_{2}^{*} & t_{1}^{*} & t_{2}^{*} & 0 & \varepsilon_{2} & 0 \\
t_{1}^{*} & t_{2}^{*} & t_{2}^{*} & 0 & 0 & \varepsilon_{2}
\end{array}\right)\left(\begin{array}{l}
a_{1} \\
a_{2} \\
a_{3} \\
b_{1} \\
b_{2} \\
b_{3}
\end{array}\right)=E\left(\begin{array}{l}
a_{1} \\
a_{2} \\
a_{3} \\
b_{1} \\
b_{2} \\
b_{3}
\end{array}\right)
$$

where $a_{\mathrm{i}}$ and $b_{\mathrm{i}}$ are the coefficients of the normalized $p_{\mathrm{z}}$ orbitals of the top and bottom $\mathrm{Si}$ atoms respectively in the linear combinations of the wave function (see Fig. $4 \mathrm{c}$ for the labeling of the atom sites). The resulting band gap $\Delta=\left|t_{1}-t_{2}\right|$, which is independent of $\varepsilon_{1}$ and $\varepsilon_{2}$. Therefore, the band gap opening at the Dirac point for the $\mathrm{AMSi}_{3 \mathrm{x}}$ monolayer is completely attributed to breaking of the bond symmetry.

Back to Fig. 3c, the band gaps of the $\mathrm{LiSi}_{6}$ and $\mathrm{LiSi}_{18}$ monolayers are found apparently larger than those of other AM-covered monolayers at the same coverage. This difference can also be attributed to the stronger bond imbalance by Li adsorption. Apart from the ionic interaction between the AM atoms and silicene, the AM atoms have a dative covalent contribution, which adds extra imbalance to the bonds. The covalent contribution is the most significant for $\mathrm{Li}$ and decreases from $\mathrm{Li}$ to $\mathrm{Cs}^{29}$. As a result, the bond symmetry is broken the most, and the magnitude of $\left|t_{1}-t_{2}\right|$ is the maximized by $\mathrm{Li}$ adsorption. Comparing Fig. $4 \mathrm{a}$ with $4 \mathrm{~b}$, we can see that the potential discrepancy of the $\mathrm{LiSi}_{6}$ monolayer between the bonds in a hexagon with and without the AM atoms is greater than that of the $\mathrm{CsSi}_{6}$ monolayer. What we want to emphasize here is that the Kekule

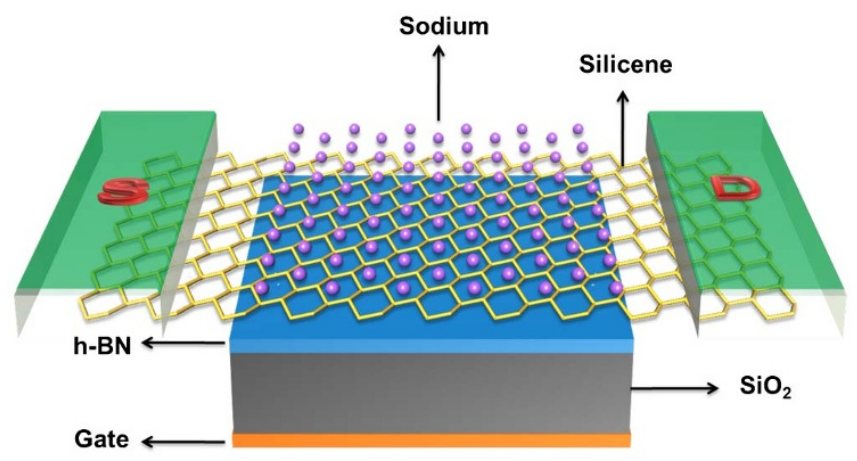

Figure $5 \mid$ Schematic model of the FET based on the Na-covered silicene. The channel is $113.8 \AA$ long, and the electrodes are composed of semiinfinite silicene.

distortion happens only if $n=3 x$. For instance, no band gap has been experimentally observed in $\mathrm{Na}$-covered graphene sample $\left(\mathrm{NaC}_{8}\right)$ grown on $\mathrm{SiC}$ substrate ${ }^{32}$.

We can also interpret the "three" dependence of the band gap creation in silicene in the $k$-space. The AM atoms serve as two-dimensional periodic potential, and certain distribution pattern of the AM atoms imposes various periodic boundary conditions into the system. In our case of the $\mathrm{AMSi}_{3 \mathrm{x}}$ monolayer, the AM atoms expand the lattice period and shrink the period in the corresponding Brillouin zone (BZ). As shown in Fig. $4 \mathrm{~d}$, the reduced $\mathrm{BZ}$ of the $\mathrm{AMSi}_{6}$ monolayer is colored green, and the purple one indicates that of silicene. Both the two inequivalent Dirac points of silicene $K_{\mathrm{s}}$ and $K_{\mathrm{S}}^{\prime}$ are mapped on the $\Gamma$ point by the reciprocal unit vector $\vec{G}_{1}$ or $\vec{G}_{2}$ in the reduced BZ (An extra $\pi / 6$ rotation with respect to the original $\mathrm{BZ}$ of silicene is also included for the $\mathrm{AMSi}_{6}$ monolayer). There is interaction between $K_{\mathrm{s}}$ and $K_{S}^{\prime}$, and thus the intervalley hopping matrix element is normally nonzero, which opens a band gap in silicene.

Effective masses of electrons $\left(m_{\mathrm{e}}\right)$ of the AM-covered silicene are slightly anisotropic, with a discrepancy less than $5 \%$. The anisotropic propagation of charge carriers is related to their chiral nature. An similar anisotropic behavior of the Dirac fermions under periodic potential in graphene has already been reported by Louie et $a l^{33}$. The $m_{\mathrm{e}}$ averaged over the different directions is provided in Fig. $3 \mathrm{~d}$. The $m_{\mathrm{e}}$ values generally increase with the coverage except $\mathrm{Li}$, with values ranging from 0.02 to $0.20 m_{0}$ ( $m_{0}$ is the free electron mass). The values of $m_{\mathrm{e}}$ are more dispersed at $N=5.6$ and $16.7 \%$ than at $N=3.1,12.5$, and $50.0 \%$ because the band structures near the Dirac point at the former two coverages are dominated by the AM-sensitive breaking of bond symmetry while those at the latter three coverages originate from the AM-insensitive breaking of sublattice symmetry. In light of the fact that suspended bilayer graphene has an effective mass of $m_{\mathrm{e}}=0.03 m_{0}$ and carrier mobility of $\mu=2 \times 10^{5} \mathrm{~cm}^{2} / \mathrm{V} \cdot \mathrm{s}^{34}$, the carrier mobility $\mu$ in AM-covered silicene is estimated in the range of $2 \times 10^{5} \sim 10^{6} \mathrm{~cm}^{2} / \mathrm{V} \bullet$ s, assuming that its scattering time $\tau$ is similar to that of suspended graphene. Since the carrier mobility of bilayer graphene is not significantly degraded by $\mathrm{Al}$ and molecule single-side adorption $^{8,25,35}$, the scattering time $\tau$ in silicene is probably not significantly decreased by the AM adsorption. We have also calculated the effective mass of holes $\left(m_{\mathrm{h}}\right)$ and found the average $m_{\mathrm{h}}$ is $1 \% \sim 9 \%$ less than the average $m_{\mathrm{e}}$, and the asymmetry between electrons and holes becomes more obvious with the increasing coverage.

Transport property. To assess the transport performance of the AMcovered silicene, we further simulate an FET out of the Na-covered silicene with $N=50 \%$. As shown in Fig. 5, the central region of the simulated FET is a $\mathrm{NaSi}_{2}$ monolayer with a length of $113.8 \AA$. The electrodes are composed of semi-infinite silicene. The dielectric region, with a thickness of $7 \AA$, consists of a $\mathrm{SiO}_{2}$ substrate and h$\mathrm{BN}$ buffer layers to preserve the high carrier mobility of silicene ${ }^{19}$. 
Since the h-BN and $\mathrm{SiO}_{2}$ share similar dielectric constant $(\varepsilon)$, we use a homogenous $\varepsilon=3.9$ to the dielectric region in the transport calculation. A bottom gate is placed below the dielectric region.

When $V_{\mathrm{g}}=0 \mathrm{~V}$, the transport gap is located below $E_{f}$ and about $0.7 \mathrm{eV}$ in size with the SZ basis set (Fig. 6a), a value about 1.5 times the band gap of $0.45 \mathrm{eV}$ obtained with the DNP basis set. The SZ basis set that used in the device simulation may be inefficient in describing some systems and usually overestimates the transport gap $^{19}$. Application of a larger SZP basis set results to an improved transport gap of $0.40 \mathrm{eV}$ (Supplementary Fig. S5). Within the bias window, there is a broad transmission peak, which is responsible for the on-state current of $236 \mu \mathrm{A} / \mu \mathrm{m}$. As $V_{\mathrm{g}}$ decreases to $-18 \mathrm{~V}, E_{f}$ shifts downward and becomes closer to the transport gap (See right insets of Fig. 6a). Correspondingly, the peak in the transmission window becomes narrower and the current decreases to $18.0 \mu \mathrm{A} /$ $\mu \mathrm{m}$. When $V_{\mathrm{g}}=-30 \mathrm{~V}, E_{f}$ is located in the opened gap of the Dirac cone, and the transmission probability nearly vanishes within the transmission window. As a result, an effective off-state with a tiny current of $5.45 \times 10^{-7} \mu \mathrm{A} / \mu \mathrm{m}$ is achieved.

Further support for the successful switch capability is provided by the transmission eigenstate at $E_{f}$ and $k$-point $=(0,1 / 3)$ compared between the on- and off-state, as shown in Fig. $6 \mathrm{~b}$. The transmission eigenvalue in the on-state is 0.70 ; correspondingly, the incoming wave function is slightly scattered and most of the incoming wave reaches the other lead. By contrast, the transmission eigenvalue in the off-state is merely 0.01 ; correspondingly, the incoming wave function is almost completely scattered and unable to reach the other lead.

Fig. $6 \mathrm{c}$ shows the transfer characteristics of the $\mathrm{NaSi}_{2}$ monolayer FET, typical of $n$ type doping. The on/off current ratio in the Nacovered silicene FET is as high as $4 \times 10^{8}$, which meets the requirement for high-speed logic applications. Such an on/off current ratio is impressive when compared with graphene, where the observed on/ off current ratio without degrading the electronic properties in graphene is no more than 100 at room temperature and 2000 at $20 \mathrm{~K}^{3-9}$. Very recently, the measured on-off current ratio is greatly improved to 10000 at room temperature in graphene tunneling $\mathrm{FET}^{36}$. However, the on-state current, which is one important figure of merit of an FET and determines the device operation speed, is greatly depressed in graphene tunneling FET due to its tunneling feature ${ }^{36}$. The calculated subthreshold swing from Fig. $6 \mathrm{c}$ is $S=140 \mathrm{mV} / \mathrm{dec}$, and the terminal transconductance is $g_{\mathrm{m}}=12.6 \mu \mathrm{S} / \mu \mathrm{m}$, ensuring a quick current response to the variation in $V_{\mathrm{g}}$ value.

\section{Discussion}

The h-BN buffer layer is shown to prevent silicene from undesired bonding with the $\mathrm{SiO}_{2}$ substrate, keeping the high carrier mobility in silicene $^{19}$. We estimate the effect of the h-BN buffer layer on the electronic structure of a freestanding $\mathrm{NaSi}_{8}$ monolayer. The addition of the h-BN sheet seldom disturbs the geometry of the freestanding $\mathrm{Na}$-covered silicene since the relatively weak interaction associated with a large distance of $d_{2}=3.6 \AA$ (Fig. 1f). As indicated by the redline in Fig. $3 c$, the band structure in the vicinity of the Dirac point is marginally perturbed by the h-BN buffer layer. Far away from the Dirac point, there are some slight shifts of the energy bands near the $\Gamma$ point, which are of little influence upon the transport performance. The h-BN layers induce slightly more asymmetry to the sublattices of the Na-covered silicene, involving a tiny band gap increment of $0.024 \mathrm{eV}$. On the other hand, it slightly slows down the slope of the bands near the Dirac points, leading to a slight increase of $m_{\mathrm{e}}$ from $4.6 \times 10^{-2}$ to $5.5 \times 10^{-2} \mathrm{~m}_{0}$.

We have carried out a calculation including the spin-orbit coupling (SOC) effects in the $\mathrm{NaSi}_{8}$ monolayer. The energy differences between the most stable and metastable states are nearly unchanged (increased by $0.03 \%$ ). The SOC effects is predicted to cause a semimetal to semiconductor transition in pure silicene with a direct gap of $\sim 15 \mathrm{meV}$ in the Dirac point and tends to be larger with heavier a

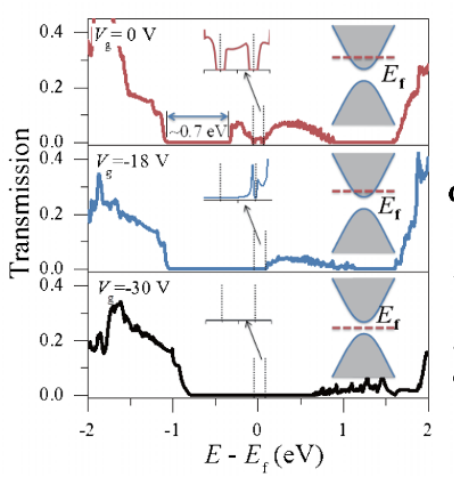

b $\quad V_{\mathrm{g}}=0 \mathrm{~V}$
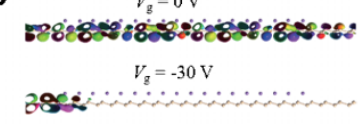

Phase (radians)

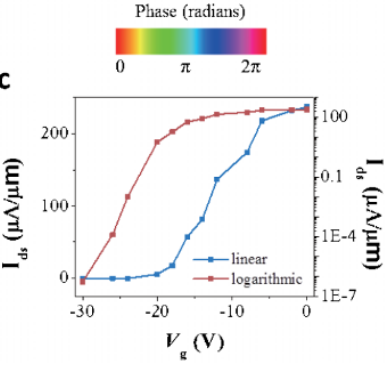

Figure $6 \mid$ Device performance of the Na-covered $(N=50 \%)$ silicene FET. The bias voltage is set to $0.1 \mathrm{~V}$. (a) Transmission spectra with $V_{\mathrm{g}}=0,-18$ and $-30 \mathrm{~V}$, respectively. The vertical dashed-line indicates the bias window. The details in the bias window are provided in the left insets. The right insets are sketches illustrating the relative positions of $E_{\mathrm{f}}$ and the gap manipulated by $V_{\mathrm{g}}$. (b) Transmission eigenstates (at $E=E_{f}$ and $k$-point $=$ $(0,1 / 3))$ of the on-state $\left(V_{\mathrm{g}}=0 \mathrm{~V}\right)$ and off-state $\left(V_{\mathrm{g}}=-30 \mathrm{~V}\right)$. The isovalue is 0.2 a.u. (c) Transfer characteristic in linear (left axis) and logarithmic scales (right axis).

buckling ${ }^{37,38}$. As shown in Supplementary Fig. S3, the band structures of a $\mathrm{NaSi}_{8}$ monolayer with and without the inclusion of the SOC effects are nearly the same. The band gap increases by only $1.2 \mathrm{meV}$ after the inclusion of the SOC effects.

Many-body effects may significantly enlarge the band gap in semiconductors, especially in the low dimensional systems ${ }^{39}$. We also test many-body effects on the opened band gap in AM-covered silicene. Supplementary Fig. S4 shows the band structures of the $\mathrm{NaSi}_{2}$ monolayer with and without the inclusion of the many-body effects. The $\pi^{*}$ state around the Dirac points shifts upward, and the band gap increases slightly from 0.34 to $0.38 \mathrm{eV}$ upon the inclusion of the many-body effects. The slight increase in the band gap is attributed to the fact that the AM-covered silicene is a metal in fact, and the opened gap in the Dirac cone is $0.5 \sim 0.7 \mathrm{eV}$ lower than $E_{f}$. When $E_{f}$ is shifted to the band gap region by the gate (off-state), the increasing magnitude of the band gap upon the inclusion of the many-body effects should be much larger. In the device simulation, the unconsidered many-body effects may cancel the artificial increment error of the transport gap by using the smaller SZ basis set ${ }^{19}$.

We also investigate the transport properties of the FET composing of a $\mathrm{KSi}_{2}$ or $\mathrm{LiSi}_{2}$ monolayer channel (Supplementary Fig. S6). When the $\mathrm{SZ}$ basis set is used, the transport gaps in the $\mathrm{KSi}_{2}$ and $\mathrm{LiSi}_{2}$ monolayer FET are calculated to be 0.7 and $0.6 \mathrm{eV}$, respectively, which are comparable with that in the $\mathrm{NaSi}_{2}$ monolayer FET. The calculated on/off current ratio of the $\mathrm{KSi}_{2}$ monolayer FET is also up to $6 \times 10^{6}$, suggestive of great potential of a $\mathrm{KSi}_{2}$ monolayer in a high-performance FET.

In summary, our $a b$ initio calculations demonstrate that AM surface adsorption is able to create a sizable band gap $(>0.4 \mathrm{eV})$ in silicene without degrading its extremely high mobility. Different from the band gap oscillating with the coverage in single layer graphene, the band gap opened in AM-covered silicene is dual-protected by the breaking of sublattice and bond symmetry and generally increases with the coverage. The high on/off ratio of $10^{8}$ obtained in the simulated AM-covered silicene FET suggests potential advantage of the AM-covered silicene for low-power consumption and high-speed logic devices. We anticipate experimental realization of the AM surface adsorption on silicene and outstanding performance in AM-covered silicene FETs.

\section{Methods}

First, we perform DFT calculations within the generalized gradient approximation (GGA) to the Perdew-Wang (PW91) exchange-correlation functional ${ }^{40}$. The geometry 
optimizations and electronic structure calculations are performed with the all-electron double numerical atomic orbital plus polarization (DNP) basis set ${ }^{41}$, as implemented in the $\mathrm{Dmol}^{3}$ package ${ }^{42}$. A DFT-D semi-empirical correction is applied with the PW91 functional to account for the dispersion interaction; the dipole correction is used to eliminate the artificial dipole moments interaction between the periodic images along the $z$-direction. The Monkhorst-Pack $k$-point mesh $^{43}$ is sampled with a separation of about $0.01 \AA^{-1}$ in the Brillouin zone. The geometry optimization is carried out until the maximum force on each atom is less than $10^{-3} \mathrm{eV} / \mathrm{A}$. In order to include the SOC and many-body effects of AM adsorption, we perform some further tests (For details, see Supplementary method). In a further step, the transport properties of the gated twoprobe model is established by the DFT coupled with the nonequilibrium Green's function (NEGF) method, as implemented in the ATK 11.8 package ${ }^{44,45}$. We employ the single-zeta (SZ) basis set during the device simulation, and a test with single-zeta plus polarization (SZP) basis set is also carried out for comparison. The MonkhorstPack $k$-point meshes for the central region and electrodes are sampled with $1 \times 50 \times 1$ and $1 \times 50 \times 50$ separately. The temperature is set to $300 \mathrm{~K}$. Following the LandauerBüttiker formalism ${ }^{46}$, the current $I$ is obtained by

$$
I\left(V_{\mathrm{g}}, V_{\mathrm{b}}\right)=\frac{2 e}{h} \int_{-\infty}^{+\infty}\left\{T\left(E, V_{\mathrm{g}}, V_{\mathrm{b}}\right)\left[f\left(E-\mu_{\mathrm{L}}\right)-f\left(E-\mu_{\mathrm{R}}\right)\right]\right\} \mathrm{d} E,
$$

where $T\left(E, V_{\mathrm{g}}, V_{\mathrm{b}}\right)$ is the transmission probability at a given gate voltage $V_{\mathrm{g}}$ and bias voltage $V_{\mathrm{b}}, f(E)$ is the Fermi-Dirac distribution function, and $\mu_{\mathrm{L}} / \mu_{\mathrm{R}}$ is the electrochemical potentials of the left/right electrode $\left(\mu_{\mathrm{R}}-\mu_{\mathrm{L}}=\mathrm{e} V_{\mathrm{b}}\right)$. The electrostatic responses of $V_{\mathrm{b}}$ and $V_{\mathrm{g}}$ are calculated by solving the Poisson equation self-consistently. The Neumann condition is used on the boundaries of the direction vertical to the silicene plane. On the surfaces connecting the electrodes and the central region, we employ Dirichlet boundary condition to ensure the charge neutrality in the source and the drain region.

1. Novoselov, K. S. et al. Electric field effect in atomically thin carbon films. Science 306, 666-669 (2004)

2. Schwierz, F. Graphene transistors. Nat. Nanotech. 5, 487-496 (2010).

3. Quhe, R. G. et al. Tunable and sizable band gap of single-layer graphene sandwiched between hexagonal boron nitride. NPG Asia Mater. 4, e6 (2012).

4. Yavari, F. et al. Tunable bandgap in graphene by the controlled adsorption of water molecules. Small 6, 2535-2538 (2010).

5. Tian, X. Q., Xu, J. B. \& Wang, X. M. Band gap opening of bilayer graphene by $\mathrm{F}_{4}$ TCNQ molecular doping and externally applied electric field. J. Phys. Chem. B 114, 11377-11381 (2010)

6. Castro, E. V. et al. Biased bilayer graphene: Semiconductor with a gap tunable by the electric field effect. Phys. Rev. Lett. 99, 216802 (2007).

7. Zhang, W. J. et al. Opening an electrical band gap of bilayer graphene with molecular doping. ACS Nano 5, 7517-7524 (2011).

8. Park, J. et al. Single-gate bandgap opening of bilayer graphene by dual molecular doping. Adv. Mater. 24, 407-411 (2012).

9. Xia, F. N., Farmer, D. B., Lin, Y.-M. \& Avouris, P. Graphene field-effect transistors with high on/off current ratio and large transport band gap at room temperature. Nano Lett. 10, 715-718 (2010)

10. Cahangirov, S., Topsakal, M., Aktürk, E., Şahin, H. \& Ciraci, S. Two- and onedimensional honeycomb structures of silicon and germanium. Phys. Rev. Lett. 102, 236804 (2009).

11. Guzmán-Verri, G. G. \& Lew Yan Voon, L. C. Electronic structure of silicon-based nanostructures. Phys. Rev. B 76, 075131 (2007).

12. Vogt, P. et al. Silicene: Compelling experimental evidence for graphenelike twodimensional silicon. Phys. Rev. Lett. 108, 155501 (2012).

13. Lin, C. L. et al. Structure of silicene grown on Ag(111). Applied Physics Express 5, 3 (2012).

14. Jamgotchian, H. et al. Growth of silicene layers on $\operatorname{Ag}(111)$ : Unexpected effect of the substrate temperature. J. Phys. Condens. Mat. 24, 7 (2012).

15. Le Lay, G. et al. Physics and chemistry of silicene nano-ribbons. Appl. Surf. Sci. 256, 524-529 (2009)

16. De Padova, P. et al. Evidence of graphene-like electronic signature in silicene nanoribbons. Appl. Phys. Lett. 96, 261905 (2010)

17. Feng, B. J. et al. Evidence of silicene in honeycomb structures of silicon on Ag(111). Nano Lett. 12, 3507-3511 (2012).

18. Fleurence, A. et al. Experimental evidence for epitaxial silicene on diboride thin films. Phys. Rev. Lett. 108, 245501 (2012)

19. Ni, Z. Y. et al. Tunable bandgap in silicene and germanene. Nano Lett. 12, 113-118 (2012).

20. Gao, N., Zheng, W. T. \& Jiang, Q. Density functional theory calculations for twodimensional silicene with halogen functionalization. Phys. Chem. Chem. Phys. 14, 257-261 (2012).

21. Osborn, T. H., Farajian, A. A., Pupysheva, O. V., Aga, R. S. \& Lew Yan Voon, L. C. Ab initio simulations of silicene hydrogenation. Chem. Phys. Lett. 511, 101-105 (2011).

22. Houssa, M. et al. Electronic properties of hydrogenated silicene and germanene. Appl. Phys. Lett. 98, 223107 (2011).

23. Elias, D. C. et al. Control of graphene's properties by reversible hydrogenation: Evidence for graphane. Science 323, 610-613 (2009).

24. Jaiswal, M. et al. Controlled hydrogenation of graphene sheets and nanoribbons. ACS Nano 5, 888-896 (2011)
25. Szafranek, B. N., Schall, D., Otto, M., Neumaier, D. \& Kurz, H. High on/off ratios in bilayer graphene field effect transistors realized by surface dopants. Nano Lett. 11, 2640-2643 (2011)

26. Pan, L. et al. First-principles study of monolayer and bilayer honeycomb structures of group-IV elements and their binary compounds. Phys. Lett. A 375, 614-619 (2010)

27. Sahin, H. et al. Monolayer honeycomb structures of group-IV elements and III-V binary compounds: First-principles calculations. Phys. Rev. B 80, 155453 (2009).

28. Chan, K. T., Neaton, J. B. \& Cohen, M. L. First-principles study of metal adatom adsorption on graphene. Phys. Rev. B 77, 235430 (2008)

29. Clotet, A., Ricart, J. M., Rubio, J. \& Illas, F. Nature of bonding of alkali metals to Si(111). Phys. Rev. B 51, 1581-1592 (1995).

30. Yang, J. W., Lee, G., Kim, J. S. \& Kim, K. S. Gap opening of graphene by dual $\mathrm{FeCl}_{3}$ acceptor and K-donor doping. J. Phys. Chem. Lett. 2, 2577-2581 (2011).

31. Farjam, M. \& Rafii-Tabar, H. Energy gap opening in submonolayer lithium on graphene: Local density functional and tight-binding calculations. Phys. Rev. B 79, 045417 (2009).

32. Hwang, C. G. et al. Stability of graphene band structures against an external periodic perturbation: Na on graphene. Phys. Rev. B 79, 115439 (2009).

33. Park, C.-H., Yang, L., Son, Y.-W., Cohen, M. L. \& Louie, S. G. Anisotropic behaviours of massless Dirac fermions in graphene under periodic potentials. Nat. Phys. 4, 213-217 (2008).

34. Bolotin, K. I. et al. Ultrahigh electron mobility in suspended graphene. Solid State Commun 146, 351-355 (2008).

35. Miyazaki, H., Tsukagoshi, K., Kanda, A., Otani, M. \& Okada, S. Influence of disorder on conductance in bilayer graphene under perpendicular electric field. Nano Lett. 10, 3888-3892 (2010).

36. Britnell, L. et al. Field-effect tunneling transistor based on vertical graphene heterostructures. Science 335, 947-950 (2012).

37. Liu, C.-C., Feng, W. X. \& Yao, Y. G. Quantum spin hall effect in silicene and twodimensional germanium. Phys. Rev. Lett. 107, 076802 (2011).

38. Liu, C.-C., Jiang, H. \& Yao, Y. G. Low-energy effective Hamiltonian involving spin-orbit coupling in silicene and two-dimensional germanium and tin. Phys. Rev. B 84, 195430 (2011).

39. Wirtz, L., Marini, A. \& Rubio, A. Excitons in boron nitride nanotubes: Dimensionality effects. Phys. Rev. Lett. 96, 4 (2006).

40. Perdew, J. P. et al. Atoms, molecules, solids, and surfaces: Applications of the generalized gradient approximation for exchange and correlation. Phys. Rev. B 46, 6671-6687 (1992).

41. Delley, B. An all-electron numerical-method for solving the local density functional for polyatomic-molecules. J. Chem. Phys. 92, 508-517 (1990).

42. Delley, B. From molecules to solids with the $\mathrm{DMol}^{3}$ approach. J. Chem. Phys. 113, 7756-7764 (2000).

43. Monkhorst, H. J. \& Pack, J. D. Special points for brillouin-zone integrations. Phys. Rev. B 13, 5188-5192 (1976).

44. Brandbyge, M., Mozos, J. L., Ordejon, P., Taylor, J. \& Stokbro, K. Density-functional method for nonequilibrium electron transport. Phys. Rev. B 65, 17 (2002).

45. José, M. S. et al. The SIESTA method for ab initio order-N materials simulation. J. Phys. Condense. Mat. 14, 2745 (2002).

46. Datta, S. Electronic Transport in Mesoscopic Systems, (Cambridge University Press, Cambridge, England, 1995).

\section{Acknowledgements}

This work was supported by the NSFC (Grant Nos. 10774003 and 11274016), National 973 Projects (Nos. 2007CB936200 and 2013CB932604, MOST of China), Fundamental Research Funds for the Central Universities, National Foundation for Fostering Talents of Basic Science (No. J1030310/No.J1103205), and Program for New Century Excellent Talents in University of MOE of China.

\section{Author contributions}

The idea was conceived by J. L. The DFT electronic band calculation ( $\mathrm{Dmol}^{3}$ part) was performed by R. Q., Y. W., and Z. N., and the device simulation was performed by R. Q. J. Z., H. L., C. X. The DFT calculation involving the SOC effects was tested by Q. L. The $G W$ correction was performed by R. F. The data analyses were performed by J. L., Z. G., D. Y., and R. Q. This manuscript was written by R. Q., Q. L., R. F., and J. L. All authors contributed to the preparation of this manuscript.

\section{Additional information}

Supplementary information accompanies this paper at http://www.nature.com/ scientificreports

Competing financial interests: The authors declare no competing financial interests.

License: This work is licensed under a Creative Commons

Attribution-NonCommercial-ShareAlike 3.0 Unported License. To view a copy of this license, visit http://creativecommons.org/licenses/by-nc-sa/3.0/

How to cite this article: Quhe, R. et al. Tunable and sizable band gap in silicene by surface adsorption. Sci. Rep. 2, 853; DOI:10.1038/srep00853 (2012). 